\title{
PROCESSO DE TOMADA DE DECISÃO QUANTO À POLÍTICA DE MANUTENÇÃO
}

\section{DECISION-MAKING PROCESS FOR MAINTENANCE POLICY}

\author{
Thiago de Almeida Rodrigues ${ }^{1}$; Robson Seleme ${ }^{2}$; Marcelo Gechele Cleto ${ }^{3}$ \\ ${ }^{1}$ Universidade Federal do Paraná (UFPR) - Curitiba - PR - Brasil \\ trodrigues.almeida@gmail.com \\ ${ }^{2}$ Universidade Federal do Paraná (UFPR) - Curitiba - PR - Brasil \\ robsonseleme@hotmail.com \\ ${ }^{3}$ Universidade Federal do Paraná (UFPR) - Curitiba - PR - Brasil \\ mgcleto@ufpr.br
}

\begin{abstract}
Resumo
As organizações atuam em cenários cada vez mais complexos e mutáveis, sendo influenciadas por fatores externos e internos que impactam nas tomadas de decisão. Entre estas, a definição de qual política de manutenção aplicar a determinado equipamento, buscando reduzir os custos e aumentar a eficiência, é de grande importância. A partir da necessidade de um processo estruturado que identifique as variáveis mais relevantes e detalhe as atividades que devem ser consideradas, definiu-se como objetivo elaborar um processo que auxilie na tomada de decisão quanto a qual política de manutenção aplicar a determinado equipamento. No processo proposto foram consideradas cinco análises para a tomada de decisão, entre elas a análise de custos, segurança, recursos e viabilidade, riscos e meio ambiente, onde cada uma poderá resultar em três possíveis políticas de manutenção sugeridas, a manutenção corretiva, preventiva ou preditiva. $O$ resultado desta pesquisa foi um macro processo estruturado de tomada de decisão quanto à política de manutenção; é importante ressaltar que o processo proposto deve ser alinhado às especificidades da organização, logo, o mesmo serve como referência, levantando questionamentos relevantes à tomada de decisão e direcionando a organização.
\end{abstract}

Palavras-chave: manutenção; processo; tomada de decisão.

\section{Introdução}

A mecanização de diversas atividades do meio empresarial, buscando obter melhores resultados operacionais em um mercado de constantes mudanças, fez com que a manutenção também evoluísse. Essas mudanças são consequências dos projetos mais complexos, aumento do número e diversidade de itens físicos como instalações e equipamentos, novos enfoques sobre a organização da manutenção e a gestão da manutenção como fator para obter melhores resultados (KARDEC; NASCIF, 2013). 
A manutenção influencia em diversos setores, estando também relacionada diretamente com a segurança, meio ambiente, custos e restrições de orçamento, utilização de mão de obra, resultados obtidos, entre outros, o que torna estimar o melhor conjunto de políticas de manutenção para os diferentes modos de falha uma tarefa difícil e complexa (BERTOLINI; BEVILACQUA, 2006). Porém o reconhecimento de manutenção como potencial gerador de resultados é recente (WAEYENBERGH; PINTELON, 2002). Portanto, um programa de manutenção adequado deve definir diferentes estratégias de manutenção para máquinas diferentes (WANG; CHU; WU, 2007).

O problema que a pesquisa busca solucionar é referente à dificuldade em definir qual política de manutenção aplicar, considerando os diversos fatores que influenciam nesta tomada de decisão. Com isso, a hipótese levantada é utilizar um processo estruturado que auxilie a mesma. Devido às diversas variáveis que integram o meio organizacional, a complexidade referente à tomada de decisão assim como a rápida mudança nos cenários da empresa justificam a necessidade de um processo que facilite a análise e decisão de qual política de manutenção aplicar, englobando as características mais relevantes.

Se fosse possível, todas as empresas utilizariam as melhores técnicas de manutenção, reduzindo drasticamente as falhas em equipamentos e aumentando a disponibilidade. Porém essa não é a realidade das organizações a nível mundial, que muitas vezes não tem recursos para investir, ou até mesmo abandonam o setor de manutenção. Portanto, o objetivo geral desta pesquisa é elaborar um processo para auxiliar a tomada de decisão quanto a qual política de manutenção aplicar por meio da análise de processos anteriores do estado da arte. Entre os objetivos específicos estão realizar um levantamento bibliográfico com conhecimento recente, confiável e de aceitação no meio acadêmico referente às técnicas de gestão da manutenção, apresentar as políticas de manutenção mais utilizadas, as variáveis mais relevantes envolvidas na tomada de decisão e as ferramentas de gestão e suporte, que servirão de apoio ao processo.

\section{Referencial teórico}

De acordo com a norma NBR 5462 (ASSOCIAÇÃO BRASILEIRA DE NORMAS TÉCNICAS (ABNT), 1994), a manutenção é a combinação de todas as ações técnicas e administrativas, incluindo as de supervisão, destinadas a manter ou recolocar um item em um estado no qual possa desempenhar uma função requerida. Complementando esta ideia, temos que manutenção também é uma chave para assegurar o uso eficiente do equipamento, bem como garantir uma produção eficiente (NGUYEN; YEUNG; CASTANIER, 2013). Além disto, a mesma também é vista por sua função estratégica, buscando garantir alta produtividade dos sistemas industriais e adequar-se à realidade de cada indústria (FACCIO et al, 2012). 
Contudo, o objetivo da manutenção não é somente superar falhas, mas também prever e evitar perda de receitas a nível gerencial (NGUYEN; YEUNG; CASTANIER, 2013). Na visão atual, a manutenção existe para que não haja manutenção, referindo-se à manutenção corretiva não planejada, uma vez que a maioria das atividades de manutenção podem ser evitadas (VELDMAN; WORTMANN; KLINGENBERG, 2011).

Diferentes empresas podem ter diferentes objetivos com relação à manutenção, porém na maioria dos casos, estes objetivos são divididos em quatro aspectos, como segurança, custos, valor agregado e viabilidade (WANG; CHU; WU, 2007). Para garantir que a empresa opere nas condições necessárias e atenda suas metas de produção a um custo ideal, a gestão da manutenção tem de tomar decisões conscientes sobre os objetivos e estratégias que precisam ser perseguidos (MUCHIRI et al, 2011).

Os conceitos referentes às políticas de manutenção constituem o quadro das ações específicas que serão aplicadas às instalações e é a personificação da forma como a empresa pensa sobre a função da manutenção com relação às operações. Como consequência, isto influencia todas as atividades de manutenção da empresa (WAEYENBERGH; PINTELON, 2002). Segundo o mesmo autor, a literatura fornece muitos conceitos de tipos de manutenção, oferecendo ideias interessantes e úteis, porém as empresas estão procurando o seu próprio conceito de manutenção personalizado, sendo este claro, flexível, que permita um feedback e seja revisto periodicamente, levando em conta as mudanças de cenários.

\subsection{Conceitos de manutenção}

De acordo com a NBR 5462 (ABNT, 1994), a Manutenção Corretiva é efetuada após a ocorrência de uma pane, destinada a recolocar um item em condições de executar uma função requerida. É uma manutenção não planejada, também chamada de reativa, buscando restaurar a condição inicial do equipamento (VELDMAN; WORTMANN; KLINGENBERG, 2011). A opção por esse tipo de manutenção deve levar em conta fatores econômicos, analisando se é mais barato consertar a falha ou tomar ações preventivas, haja vista que em algumas empresas não são necessários padrões de qualidade altos e as demandas de produção são pequenas (FILHO, 2008).

Segundo a NBR 5462 (ABNT, 1994), a Manutenção Preventiva é efetuada em intervalos predeterminados, ou de acordo com critérios prescritos, destinada a reduzir a probabilidade de falha ou a degradação do funcionamento de um item. A fim de reduzir a frequência dessas falhas e dos custos de correção, serviços de Manutenção Preventiva são aplicados ao longo do ciclo de vida do sistema (WU; XIE; ADAM, 2011). Consistindo em ações como substituição de um elemento, limpeza, ajuste, lubrificação, entre outros, buscam melhorar as condições dos elementos e aumentar a vida útil do mesmo, mantendo-o em condição de operação (BRIS; CHÂTELET; YALAOUI, 
2003). As ações são realizadas em intervalos predeterminados de acordo com critérios predefinidos por técnicos de manutenção, reduzindo o fator improvisação e proporcionando maior 'tranquilidade' operacional (VIANA, 2002).

A Manutenção Preditiva segundo a NBR 5462 (ABNT, 1994), permite garantir uma qualidade de serviço desejada, com base na aplicação sistemática de técnicas de análise, utilizandose de meios de supervisão centralizados ou de amostragem, para reduzir ao mínimo a Manutenção Preventiva e diminuir a Manutenção Corretiva. Da inspeção visual, que é o método mais antigo e ainda assim um dos mais poderosos e amplamente utilizados, a Manutenção Preditiva evoluiu para métodos automatizados que utilizam técnicas de processamento de sinal avançadas baseadas em reconhecimento de padrões. Quando os equipamentos começam a falhar, demonstram sinais (ruídos, vibrações, cheiro, temperatura), que podem ser detectados por operadores ou equipamentos através de inspeções (HASHEMIAN; BEAN, 2011).

Outra política da gestão da manutenção é o Total Productive Maintenance (TPM), que teve seu conceito desenvolvido inicialmente em 1971 pelo grupo Toyota no Japão, em resposta aos problemas de manutenção e suporte encontrados nos ambientes de produção (CHAN et al, 2005). Não só uma metodologia, mas sim uma filosofia, TPM tem sido descrito como uma abordagem de parceria para manutenção (SWANSON, 2001). Entre os objetivos de implantação, o TPM busca aumentar a disponibilidade dos equipamentos, sendo projetada para evitar perdas por paradas devido a falhas e ajustes, perda de velocidade, defeitos ao longo dos processos e diminuição do rendimento, maximizando a eficiência dos sistemas de produção de uma forma geral (CHAN et al, 2005). Outros objetivos apresentados por Ben-Daya (2000), são a melhora da qualidade do produto, melhora do estado de conservação e capacitação dos funcionários. Segundo o mesmo autor, estes objetivos são alcançados através de uma implantação cuidadosa dos conceitos de capacitação dos funcionários e da boa gestão dos equipamentos.

Já a manutenção centrada na confiabilidade, do inglês Reliability Centered Maintenance (RCM), é uma abordagem sistemática para projetar a Manutenção Preventiva das indústrias, tendo como objetivo preservar as funções da planta utilizando menos os recursos, ou aperfeiçoar as atividades de Manutenção Preventiva (KIANFAR; KIANFAR, 2010). Seu conceito foi originado na indústria aeronáutica na década de 70, e a partir de experiências em indústrias de energia, gás, nuclear, petróleo entre outras, demonstrou uma redução significativa dos custos da Manutenção Preventiva (RAUSAND, 1998). A metodologia RCM pode ser descrita pelas três fases seguintes: 1identificação dos itens de manutenção significativos; 2- atribuição de tarefas de Manutenção Preventiva adequadas para o equipamento; 3 - implantação e atualização das tarefas de Manutenção Preventiva (SELVIK; AVEN, 2011). Segundo o mesmo autor, para os itens que não forem classificados como significativos, a estratégia ideal é utilizar a Manutenção Corretiva. 


\subsection{Ferramentas de apoio à tomada de decisão}

\subsubsection{Diagrama de pareto e curva $\mathrm{ABC}$}

Em qualquer processo de melhoramento, vale a pena distinguir o que é importante e o que é menos importante. O propósito do Diagrama de Pareto é distinguir entre as "poucas questões vitais" e as "muitas questões triviais". A partir da análise de Pareto, podem-se classificar diferentes tipos de itens, variando sua forma de classificação e julgamento segundo a necessidade da organização. Classificam-se como itens classe ' $\mathrm{A}$ ' os $20 \%$ dos itens que representam $80 \%$ do valor total, 'B' os seguintes $30 \%$ dos que representam $10 \%$ do valor total e os demais, são classificados como 'C' (SLACK; CHAMBERS; JOHNSTON, 2009).

\subsubsection{FMEA}

A Análise de Modo de Falha, do inglês Failure Mode and Effects Analysis (FMEA) é uma metodologia que se concentra em priorizar falhas críticas para melhorar a segurança, confiabilidade e qualidade dos produtos e processos (SUBBURAMAN et al, 2010). O principal objetivo do FMEA é identificar os modos de falha potenciais, avaliar as causas e os efeitos de diferentes modos de falha do componente, e determinar o que poderia eliminar ou reduzir a chance de fracasso. Os resultados da análise podem ajudar a identificar e corrigir os modos de falha que têm um efeito prejudicial sobre o sistema e melhorar o seu desempenho durante as fases de concepção e produção (LIU; LIU; LIU, 2013).

O primeiro passo na FMEA é identificar todos os possíveis modos de falhas potenciais do produto ou do sistema por uma sessão de brainstorming. Depois disso, a análise crítica é realizada sobre esses modos de falha, utilizando uma escala numérica que varia de 1 a 10, levando em conta os fatores de risco: de ocorrência (O), severidade (S) e de detecção (D). O objetivo do FMEA é priorizar os modos de falha do produto ou sistema, a fim de atribuir os recursos limitados para os itens de risco mais graves (LIU; LIU; LIU, 2013).

\subsubsection{Modelagem de processos}

As empresas que querem se manter competitivas, devem ser capazes de adaptar seus processos de negócios rapidamente aos novos ambientes dinâmicos (ANDRADE; FIADEIRO, 2002). Para lidar com as necessidades impostas por esses fatores, a modelagem de processos se apresenta como um elemento central, por organizar e estruturar a visão da organização, tornando-se uma atividade que adiciona valor ao processo produtivo e ao produto (LOVE; GUNASEKARAN, 1997). 
Uma das linguagens utilizadas para modelar processos é o BPMN (Business Processes Modeling Notation), que orienta as pessoas quanto a estruturas do processo de negócio: qual o começo e término do processo; quais as etapas básicas que compõem o melhor caminho; se as etapas básicas são condicionais, concomitantes ou sequenciais e quais são os estados de exceção mais importantes? (NUFFEL; BACKER, 2012). O BPMN foi desenvolvido com a intenção de permitir a fácil compreensão do modelo e das representações gráficas dos processos de negócios (LA ROSA et al, 2011).

\subsection{Requisitos de análise para tomada de decisão}

\subsubsection{Custos de manutenção}

A análise do custo é um dos principais quesitos em todos os setores das organizações, logo, na manutenção não seria diferente. A confiabilidade de equipamentos industriais é pobre em um longo prazo no sentido de que se deterioram com a idade e, em algumas situações, a substituição do equipamento é economicamente conveniente (JACKSON; PASCUAL, 2008). Os custos em manutenção podem ser divididos em custos fixos e variáveis, dependendo do número de falhas. Os custos variáveis geralmente incluem os custos de transporte de componentes e uso de itens em massa; custos fixos geralmente incluem custos de recursos, tais como equipamentos de reparação (BASTEN; HEIJDEN; SCHUTTEN, 2011). A manutenção adequada de equipamentos da planta pode reduzir significativamente o custo operacional total e aumentar a produtividade da planta. Os gerentes muitas vezes consideram a manutenção uma despesa, mas abordagens mais positivas definem os trabalhos de manutenção como um centro de lucro (BERTOLINI; BEVILACQUA, 2006).

\subsubsection{Segurança e meio ambiente}

Entre vários fatores, a prática de manutenção também vai afetar a ocorrência de um acidente (ARUNRAJ; MAITI, 2010). A partir de uma perspectiva baseada em tarefas, realizar a manutenção é um risco. As operações de manutenção causam riscos às máquinas, à empresa e aos colaboradores que realizam as operações. Os riscos ocupacionais estão relacionados com, por exemplo, a montagem e desmontagem, trabalho feito com o equipamento em funcionamento, entre outros (LIND; NENONEN; KIVISTO-RAHNASTO, 2008).

Para análise de segurança e risco ao meio ambiente, é preciso identificar as falhas cujas consequências poderiam ter um impacto sobre ou comprometer o desempenho dos sistemas, segurança dos colaboradores ou riscos ao ambiente. Com base nesta informação, o componente, que é responsável pela falha, é identificado. Geralmente, um componente mais crítico é determinado 
pela capacidade de afetar a segurança, possibilidade de detectar uma falha durante a operação, impacto sobre o funcionamento e o impacto econômico (WAEYENBERGH; PINTELON, 2004).

\subsubsection{Recursos e viabilidade}

Os gerentes devem selecionar a política de manutenção mais adequada a cada equipamento ou sistema com base em diversos fatores, entre eles os recursos disponíveis. O desenvolvimento de estratégias de manutenção (combinando as possibilidades de políticas, como ações corretivas, preventivas e preditivas) deve levar em conta que os recursos são limitados e, como resultado, as atividades de manutenção devem ser imperfeitas, o que resulta que nem sempre será possível aplicar uma política de manutenção devido às restrições (TSAI; WANG; TSAI, 2004). Quando a organização não é capaz de realizar a manutenção, uma opção a ser considerada é a terceirização do serviço.

\subsubsection{Riscos em manutenção}

Um dos principais objetivos de uma estratégia de manutenção boa é a minimização dos riscos para os seres humanos e meio ambiente, causados pela falha inesperada do equipamento (KHAN; HADDARA, 2003). O processo de avaliação de risco envolve: identificação do perigo, a probabilidade e estimativa da consequência. Geralmente avaliado por comparação entre o risco calculado de uma unidade e um padrão (a partir dos critérios de aceitação), se o risco calculado exceder os critérios de aceitação, o mesmo deve ser reduzido. Um dos meios para reduzir o risco é o planejamento de manutenção eficaz, o que torna a consideração do mesmo como critério para a seleção de política de manutenção, fator crucial (ARUNRAJ; MAITI, 2010).

A metodologia de manutenção baseada no risco é projetada para estudar todos os modos de falha, determinando o risco associado aos mesmos e o desenvolvimento de uma estratégia de manutenção que minimize a ocorrência dos modos de falha de alto risco (ARUNRAJ; MAITI, 2007). Ao comparar os riscos associados a uma situação em que nenhuma ação será tomada com os riscos associados com implantação de uma ação, é possível identificar o ganho potencial e, portanto, o interesse da utilidade de uma ação (TAILLANDIER; SAUCE; BONETTO, 2011).

\subsection{Processos anteriores de decisão da política de manutenção}

Apresentando novos métodos para trabalhar com a manutenção e frameworks auxiliando na tomada de decisão, diversos autores contribuíram para o embasamento do processo proposto nesta pesquisa. Pintelon e Gelders (1992) apresentaram uma estrutura para investigação quantitativa na tomada de decisão da manutenção, propondo uma estrutura em três etapas: a modelagem da manutenção (para entender como ela interage com os outros departamentos como produção, 
marketing e finanças), o planejamento das decisões de manutenção (sem considerações sobre aspectos técnicos) e a utilização de estatística e ferramentas computacionais para estimar o comportamento e sobrevivência dos equipamentos. Além disto, a pesquisa também discute e apresenta a importância do conhecimento da organização, das práticas de manutenção, pesquisa operacional e de técnicas de gestão científica para construir modelos quantitativos adequados.

Outro processo que auxilia na tomada de decisão quanto à política de manutenção é o proposto por Waeyenbergh e Pintelon (2002), que apresentam um framework com orientações para o estabelecimento dos conceitos de manutenção, permitindo a cada empresa criar seu conceito específico, levando em consideração a informação computacional e o conhecimento da organização. Utilizando o estado da arte com relação ao RCM, TPM, BCM, entre outras ferramentas, os autores utilizam uma árvore de decisões, considerando aspectos técnicos e econômicos, respondendo questões com "sim" ou "não", decidindo qual politica de manutenção utilizar. Esta árvore está exemplificada na Figura 1. Aperfeiçoando estes conceitos, Waeyenbergh e Pintelon (2004) apresentam um framework de manutenção desenvolvido a partir de 7 etapas, conforme a Tabela 1.

Figura 1 - Árvore de tomada de decisão da Política de Manutenção

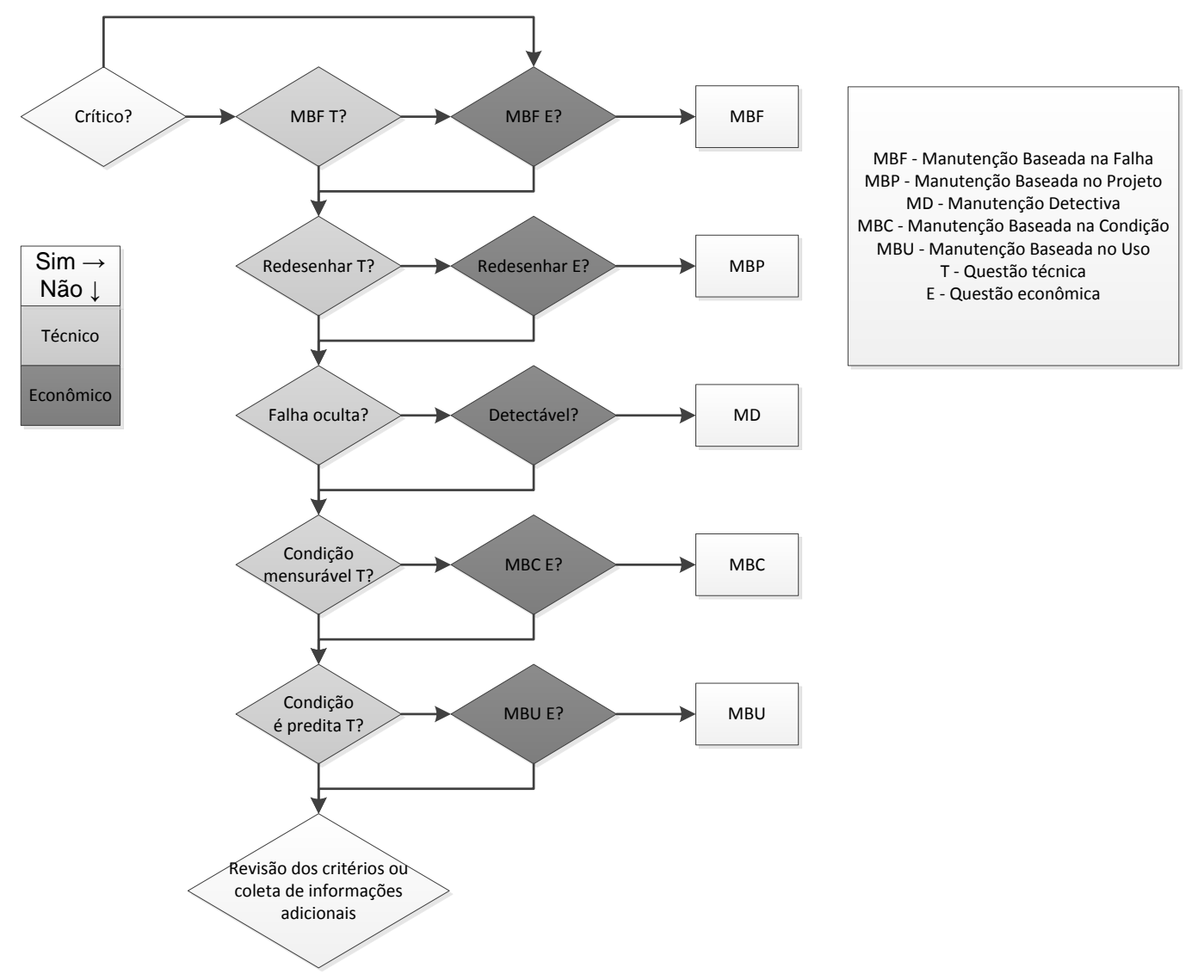

Fonte: Adaptado de Waeyenbergh e Pintelon (2002)

O primeiro módulo é o módulo de arranque, com a identificação dos objetivos e recursos. No segundo módulo, ocorre a análise técnica, onde os sistemas mais importantes e os componentes 
mais críticos serão identificados. No terceiro módulo será escolhida a política de manutenção mais adequada. O quarto módulo é o de implantação e avaliação. Dependendo do resultado, o quinto módulo, melhoria contínua, vai agir sobre os anteriores.

Tabela 1 - Quadro das 7 etapas para a política de manutenção

\begin{tabular}{lll}
\hline Etapa 1 & Identificação dos objetivos e recursos & Módulo 1 \\
\hline Etapa 2 & Seleção dos sistemas mais importantes & Módulo 2 \\
Etapa 3 & Identificação dos componentes críticos & Módulo 2 \\
Etapa 4 & Seleção da Política de Manutenção & Módulo 3 \\
& Otimização dos parâmetros da política de & \\
Etapa 5 & manutenção & Módulo 3 \\
Etapa 6 & Implantação e evolução & Módulo 4 \\
Etapa 7 & Feedback & Módulo 5 \\
\hline
\end{tabular}

Fonte: Adaptado de Waeyenbergh e Pintelon (2004)

Programas de TPM podem ser adotados por diferentes empresas e em diferentes ambientes organizacionais. De acordo McKone, Schroeder e Cua (1999) as variáveis gerenciais são mais importantes para a execução do TPM do que as variáveis organizacionais. $\mathrm{O}$ autor também alerta à importância de identificar os pontos críticos do TPM e seu impacto no desempenho organizacional, uma vez que muitas empresas deixam de investir em programas de manutenção porque gerenciam com base no orçamento e não conseguem ver as implicações estratégicas de um forte programa de manutenção.

$\mathrm{Na}$ etapa 5, correspondente ao modulo 3, ocorre a decisão quanto à politica de manutenção utilizada. Waeyenbergh e Pintelon (2004) expõem o processo de tomada de decisão, utilizando dois tipos de intervenções básicas, Manutenção Corretiva e Manutenção Preventiva, aplicando cinco distintas políticas, manutenção baseada na falha, no projeto, no uso, na condição e na detecção, sendo a política de manutenção final para ativos de uma empresa uma combinação destas alternativas.

No entanto, a fim de ser capaz de tomar decisões táticas racionais e justificáveis em relação à manutenção, é preciso ter uma ideia clara das vantagens e desvantagens de cada política de manutenção (WAEYENBERGH; PINTELON, 2004). Algumas destas etapas propostas serão utilizadas na formulação do processo para elaboração do plano de manutenção descrito nesta pesquisa.

Com uma nova metodologia para avaliação da política de Manutenção Preventiva, com base em um modelo de custo-confiabilidade que permite o uso de intervalos flexíveis de intervenções de manutenção, Lapa, Pereira e Barros (2006) utilizam técnicas de otimização (algoritmos genéticos) para procurar a política de manutenção ideal. Em sua pesquisa, para elaboração do modelo, consideram características como a probabilidade de necessidade de reparação (manutenção 
corretiva), o custo de tal reparo, tempos habituais de interrupções, custos de manutenção preventiva, o impacto da manutenção na confiabilidade dos sistemas como um todo, probabilidade de manutenção imperfeita, entre outros.

Em outro método, Muchiri et al (2011) propõe um framework conceitual que fornece diretrizes para a escolha dos indicadores de performance das funções de manutenção. Demonstrando que os indicadores de desempenho não podem ser definidos de forma isolada, mas sim ser o resultado de uma análise cuidadosa da interação entre a função de manutenção com outras funções organizacionais, a pesquisa destina-se a alinhar os objetivos de manutenção com os objetivos da fabricação e corporativos. Com base nesse framework conceitual, os indicadores do processo de manutenção e resultados de manutenção de desempenho são identificados para cada categoria.

Wang, Chu e Wu (2007) estudaram a seleção de estratégias de manutenção em empresas de manufatura. A pesquisa considera que a avaliação da manutenção para cada equipamento é uma decisão multicritério e, considerando os julgamentos imprecisos dos tomadores de decisão, o AHP (Analytic Hierarchy Process) fuzzy é utilizado para avaliação de diferentes estratégias de manutenção, buscando um mix de estratégias que melhore a disponibilidade dos equipamentos. Entre os principais critérios utilizados nesta análise, como demonstrado na Figura 2, estão a segurança, o custo, o valor agregado e a viabilidade, buscando como alternativa a utilização da manutenção corretiva, preditiva, preventiva baseada no tempo e manutenção baseada na condição.

Figura 2 - Estrutura hierárquica do AHP fuzzy

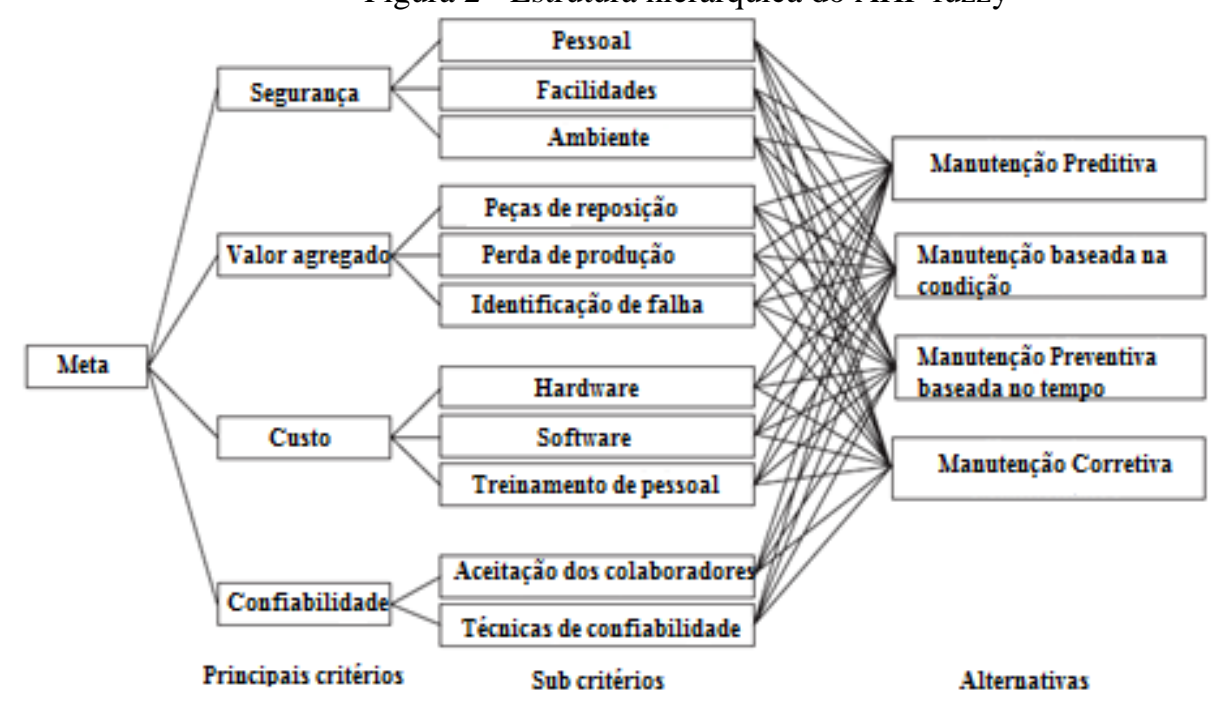

Fonte: Adaptado de Wang, Chu e Wu (2007)

Com relação aos custos de manutenção, Khans e Ingwald (2008) descrevem como um banco de dados comum pode desempenhar um papel importante para alcançar melhorias de custo benefício de desempenho da manutenção. O principal resultado apresentado é que é possível ter acesso a uma visão da produção, através de dados, e assim obter uma melhor base para a tomada de 
decisões de manutenção. Este banco de dados comum suporta o desenvolvimento de aplicações, como por exemplo, monitorar o desempenho de um processo de produção, auxiliando a decidir a política de manutenção com melhor custo benefício ou simular possíveis soluções de manutenção.

\section{Métodos e ferramentas}

Para definir o tipo de pesquisa usada, foram utilizados os critérios de classificação definidos por Vergara (2007). A pesquisa em questão se classifica quanto aos fins como uma pesquisa descritiva e, quanto aos meios, como bibliográfica.

Como meio para realização da pesquisa, um abrangente levantamento bibliográfico em fontes nacionais e internacionais de relevância foi realizado, buscando identificar o maior número de variáveis internas e externas que influenciam na tomada de decisão quanto à política de manutenção. A principal vantagem da utilização da pesquisa bibliográfica é o fato de permitir a cobertura de uma gama de fenômenos mais ampla, sendo de fundamental importância para o entendimento inicial da pesquisa (GIL, 2010).

A partir dos meios citados anteriormente, temos como fim a pesquisa descritiva, que irá expor as características de um fenômeno (VERGARA, 2007) e buscará possíveis relações entre as variáveis (GIL, 2010). Portanto, a pesquisa irá apresentar os conceitos das diferentes políticas de manutenção, determinar as variáveis e a relação entre as mesmas que influenciam na tomada de decisão, preocupando-se em observar, registrar, analisar, classificar e interpretar fatos, descrevendo os aspectos comportamentais e técnicos relevantes.

A partir das variáveis do processo levantadas, segue-se com a formulação do processo proposto para tomada de decisão, que é o foco da pesquisa. Dentro do processo geral, serão exploradas ferramentas que servirão de apoio, garantindo assim maior padronização ao longo das atividades integrantes. Ao final do processo, será aplicada uma ponderação referente aos quesitos de análise, buscando identificar qual política de manutenção aplicar.

Para justificar as atividades do processo foram utilizadas principalmente as experiências citadas na literatura, buscando uma inter-relação entre as variáveis. A natureza do estudo é definida como qualitativa, uma vez que não foram realizados estudos estatísticos. Para auxiliar a formulação do processo, utilizou-se o programa Microsoft Visio 2010 como ferramenta de modelagem, seguindo a notação do BPMN (Business Processes Management Notation) e o Business Process 
Management (BPM) como filosofia aplicada à manutenção, uma vez que busca modelar, automatizar, gerenciar e otimizar os processos de negócio (KHAN, 2004), além de ser uma coleção de atividades que transformam diferentes entradas em saídas com valor aos clientes (HAMMER; CHAMPY, 1993).

O processo proposto é um modelo para auxiliar na tomada de decisão quanto à política manutenção, ou seja, ele engloba de uma forma geral, variáveis diversas do ambiente organizacional, não especificando a um tipo de empresa. Assim sendo, cada organização que deseje aplicá-lo, deverá modificá-lo segundo suas próprias especificidades, utilizando o processo apenas como direcionamento. Outra limitação da pesquisa é o fato do processo não ter sido aplicado em uma situação real, possibilitando o dimensionamento dos resultados e possíveis comparações com outras ferramentas. Além disto, não foi utilizada uma ferramenta computacional para quantificar a decisão, o que traria maior confiabilidade ao modelo.

Quanto às vantagens, o processo auxilia as organizações levantando variáveis a serem consideradas no momento de decisão da política de manutenção, que muitas vezes são esquecidas pelos gestores, reduzindo assim os riscos e aumentando a possibilidade de acertos. Além de reunir essas variáveis e demonstrar a inter-relação entre as mesmas, o processo propõe análises e ferramentas a serem utilizadas como apoio, define responsáveis e, ao final, sugere a aplicação de uma política de manutenção a ser aplicada a determinado equipamento. O processo proposto também é simples de ser aplicado, resultando em uma resposta rápida, o que é essencial devido às mudanças inesperadas dos mercados e da organização.

\section{Processo de tomada de decisão quanto à política de manutenção}

O processo proposto para tomada de decisão quanto a qual política de manutenção aplicar a determinado grupo de equipamentos inicia-se com a 'Necessidade de Plano de Manutenção Detectada' pela empresa, como apresentado na Figura 3; para facilitar a leitura do processo, algumas atividades serão especificadas nas figuras subsequentes. Partindo de uma ideia macro, o processo descrito é top-down, tendo como responsável a Gerencia Organizacional da empresa.

A segunda etapa do processo proposto, ainda sob responsabilidade da Gerência Organizacional, é definir os objetivos da organização. O que realmente a empresa quer? Responder a esta pergunta nem sempre é uma tarefa fácil, principalmente quando envolve diversos fatores, como ocorre nas empresas globais. A empresa pretende garantir confiabilidade, reduzir custos, atender a leis de segurança e de meio ambiente? Indo além, estes objetivos podem subdividir-se em disponibilidade de planta, segurança, pessoas, entre outros. Diferentes organizações possuem objetivos distintos. Dependendo dos objetivos e da situação, fatores de ponderação, que serão comentados posteriormente, podem ser usados para salientar a importância relativa de cada objetivo 
(WAEYENBERGH; PINTELON, 2002), (WAEYENBERGH; PINTELON, 2004), (WANG; CHU; WU, 2007), (BERTOLINI; BEVILACQUA, 2006).

Figura 3 - Processo de tomada de decisão quanto a política de manutenção

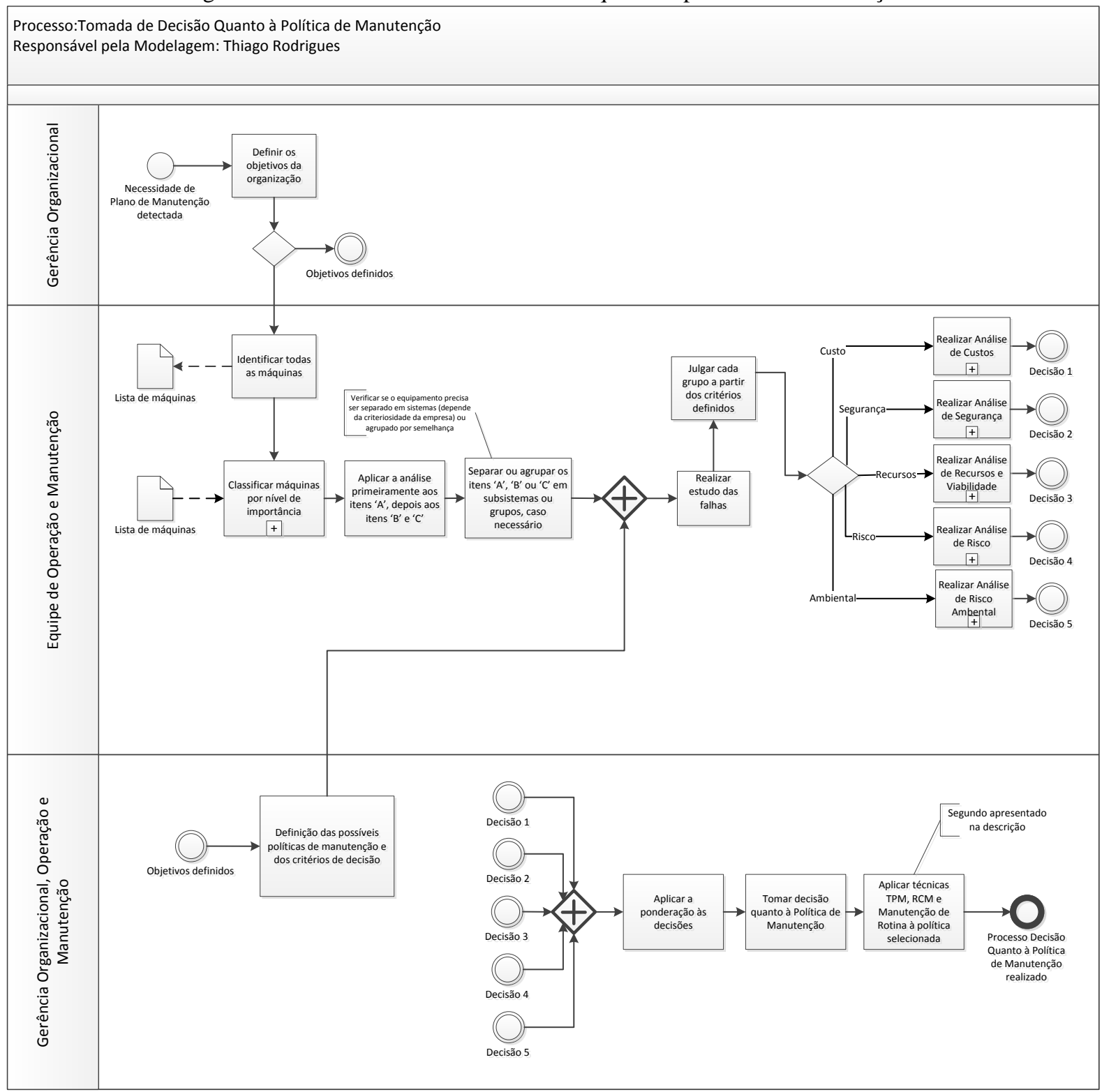

Fonte: Elaborado pelos autores

Agora que a organização já sabe o que deseja, e o que pretende atingir, a próxima etapa do processo é identificar todas as máquinas e componentes da organização. Esta atividade está sob responsabilidade da Equipe de Manutenção e Operação, que por estarem em contato direto com os equipamentos e deterem maior conhecimento sobre os mesmos, estão aptos a realizarem esta listagem. Nesta etapa fica evidente a aplicação de uma das características da filosofia TPM, com o estreitamento da relação entre Operação e Manutenção (CHAN et al, 2005).

Como cada equipamento possui características específicas, e podem afetar de diferentes maneiras a organização, dimensionar esses equipamentos e aplicar diferentes estratégias de manutenção aos mesmos é o mais adequado (WANG; CHU; WU, 2007). Esta lista de equipamentos 
pode já existir na organização, porém, a partir desta atividade, uma das saídas deverá ser um documento com esta listagem, que será utilizado na próxima atividade do processo. Estas atividades podem ser identificadas na Figura 4.

Figura 4 - Bloco das primeiras atividades do Macro processo

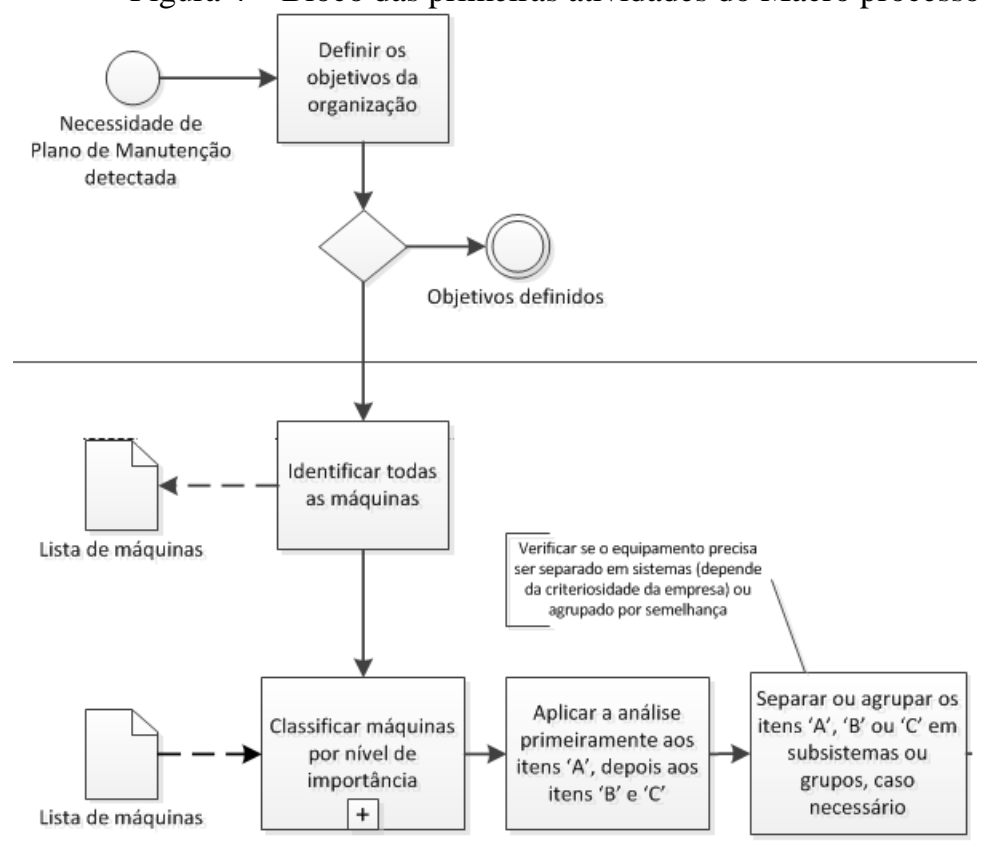

Fonte: Elaborado pelos autores

A próxima atividade do processo descrito é a classificação das máquinas por nível de importância (WAEYENBERGH; PINTELON, 2002), (WAEYENBERGH; PINTELON, 2004), (WANG; CHU; WU, 2007), (BERTOLINI; BEVILACQUA, 2006). Estes autores afirmam que para identificar o sistema mais importante, como na atividade anterior, é necessário uma interação entre produção e manutenção, porque os operadores conhecem os ambientes e as condições das instalações melhor do que todos. O objetivo desta análise é identificar os equipamentos, que a partir das falhas, possam impactar sobre o desempenho ou comprometer os sistemas, segurança e/ou meio ambiente. Como entrada para esta atividade, tem-se a lista de máquinas (resultante da atividade anterior), e utiliza-se a aplicação do método de Pareto e ABC, para classificar os equipamentos por nível de importância.

Após realizar o subprocesso de classificação dos equipamentos, os itens estão divididos em 'A', 'B' e 'C'. Na primeira etapa do processo, as atividades subsequentes serão aplicadas aos itens 'A'. Após a política de manutenção ser selecionada e aplicada para estes equipamentos, o processo continuará para os itens ' $\mathrm{B}$ ' e C'.

Um fato importante a ser considerado nos equipamentos é que o mesmo pode ser composto por uma grande quantidade de subsistemas. É como pensar em um motor de navio e realizar o mesmo tipo de manutenção em todos os componentes. Esta aplicação poderá resultar em um excesso de manutenção, grande quantidade de informação, aumento de custos, podendo até 
danificar o equipamento, realizando uma manutenção que não era necessária. Da mesma forma que um equipamento pode ser composto por diversos subsistemas, outros equipamentos podem ter grande semelhança em suas características, no tipo de reparo ou manutenção executada, podem ser utilizados pelo mesmo operador. Para os equipamentos que possuem grandes semelhanças, é recomendado agrupá-los em um único grupo, porém esta decisão deve ser tomada com uma base consistente de informação, uma vez que na etapa de análise e tomada de decisão a política de manutenção aplicada será abrangente a todo o grupo, o que também facilitará e otimizará o processo, tratando-se em empresas com uma grande quantidade de equipamentos.

Com isso, a próxima atividade do processo proposto é separar ou agrupar os itens em grupos e/ou subsistemas, caso seja necessário. Esta separação dependerá do nível de especificidade que a organização pretenda. Além do comentado anteriormente, é relevante ressaltar que a separação do equipamento em sistemas não diminui sua importância geral para a organização, ou seja, esta máquina continua sendo um item 'A', 'B' ou 'C' e será trabalhada como tal, somente será separada ou agrupada para facilitar a compreensão e a trabalhabilidade do equipamento, quanto à manutenção.

Paralelamente às atividades descritas anteriormente, sob responsabilidade do setor de manutenção e operação, tem-se a atividade de 'Definição das possíveis políticas de manutenção e dos critérios de decisão' que serão utilizados para tomada de decisão. Esta atividade é de responsabilidade conjunta entre a Gerência Organizacional, a Manutenção e a Operação, e deve ser realizada após os Objetivos da Organização serem definidos.

Agregando informações da literatura para as políticas de manutenção que são usualmente aplicadas às organizações, sugerem-se no processo as opções de Manutenção Corretiva, Manutenção Preventiva e Manutenção Preditiva (WAEYENBERGH; PINTELON, 2002), (WAEYENBERGH; PINTELON， 2004), (WANG; CHU; WU， 2007), (BERTOLINI; BEVILACQUA, 2006), (ZHAO et al, 2010), (ARUNRAJ; MAITI, 2010), (BEVILACQUA; BRAGLIA, 2000), (MARTÍNEZ et al, 2013). Buscando aproximar-se ao máximo da realidade das organizações, que ainda utilizam em grande escala Manutenção Corretiva, Preventiva, Preditiva ou nenhuma, optou-se pelas políticas de manutenção descritas acima.

Outras técnicas também estão sendo muito utilizadas nas organizações e obtendo resultados satisfatórios, como a aplicação do TPM, RCM, Manutenção de Rotina, entre outras. Porém, neste processo, considerou-se que a ferramenta TPM e RCM são mais avançadas do que as propostas, envolvendo mudança de cultura, maior envolvimento e investimento da organização, onde muitas vezes a organização escolhe um setor específico para aplicar estas técnicas, servindo como modelo (SWANSON, 2001), (NAKAJIMA, 1988), (MCKONE; SCHROEDER; CUA, 2001), (KIANFAR; KIANFAR, 2010), (SELVIK; AVEN, 2011). 
Com relação aos critérios de decisão, buscando contemplar os itens mais relevantes para a manutenção, propõe-se a realização de análise de custos, de segurança, de viabilidade e recursos e de consequências ao meio ambiente. Esta proposta baseia-se nos processos comentados anteriormente, que serviram como base para formulação deste método (JACKSON; PASCUAL, 2008), (BERTOLINI; BEVILACQUA, 2006), (WANG; CHU; WU, 2007), (WAEYENBERGH; PINTELON, 2004), (PINTELON; GELDERS, 1992), (KHAN; HADDARA, 2003), (ARUNRAJ; MAITI, 2007). Contudo, como cada empresa possui suas especificidades, cada organização deve selecionar seus critérios de decisão, assim como sua relevância. Nesse processo, utilizamos os resultados obtidos na literatura para selecionar os critérios, servindo como uma sugestão à organização. Logo, a empresa tem liberdade para adaptar o processo de acordo com suas necessidades, servindo como um guia, para auxiliar na tomada de decisão, apontando os quesitos que devem ser considerados.

Com as políticas de manutenção e os critérios de decisão definidos, assim como as máquinas classificadas por nível de importância, inicia-se a análise para posterior tomada de decisão. Deve-se iniciar pelos itens ' $\mathrm{A}$ ', como comentado anteriormente.

Como sugerem Bertolini e Bevilacqua (2006), sob responsabilidade da Manutenção e Operação, deve-se realizar um estudo com as possíveis falhas nos equipamentos. Essa listagem deve contemplar os equipamentos, seus componentes, o efeito que a falha pode causar e suas respectivas causas prováveis. Nesta etapa, não é necessário grande detalhamento dos efeitos e causas da falha, tendo a listagem como dimensionamento para as posteriores análises. Seguindo os preceitos do mesmo autor, o estudo da falha também deve contemplar uma análise das possíveis ações Preventivas e Preditivas para a falha. Indo além, propomos que ações de Manutenção Corretiva sejam inseridas. O resultado desta atividade será de grande ajuda nas análises posteriores, auxiliando os responsáveis a obterem uma visão mais ampla do problema e das possíveis soluções.

Após realizado o estudo das falhas, o processo segue com a atividade de julgar cada grupo segundo os critérios definidos anteriormente. Como citado, os critérios para análise sugeridos no processo foram análise de custos, segurança, risco, recursos e viabilidade e impacto ao meio ambiente. A organização deve julgar estes critérios, ou outros que considerarem relevantes, sempre obtendo como resultado de cada análise alguma política de manutenção a ser aplicada. Como exemplo tem-se que, ao analisar custos, define-se que a melhor política de manutenção é a corretiva, contudo, ao analisar riscos, define-se que a melhor opção é a manutenção preventiva. A partir dessas respostas, o processo seguirá para a tomada de decisão.

As próximas atividades são as análises dos critérios, como segue no modelo da Figura 5. A partir destas análises, poderá ser tomada a decisão de qual política de manutenção aplicar a determinado equipamento. As últimas atividades do processo são destinadas à tomada de decisão, 
tendo como base as análises realizadas anteriormente. A atividade de 'Aplicar ponderação às decisões' só poderá iniciar com todas as análises realizadas. O bloco final do Macro Processo é apresentado na Figura 6.

Figura 5 - Bloco das análises do Macro Processo

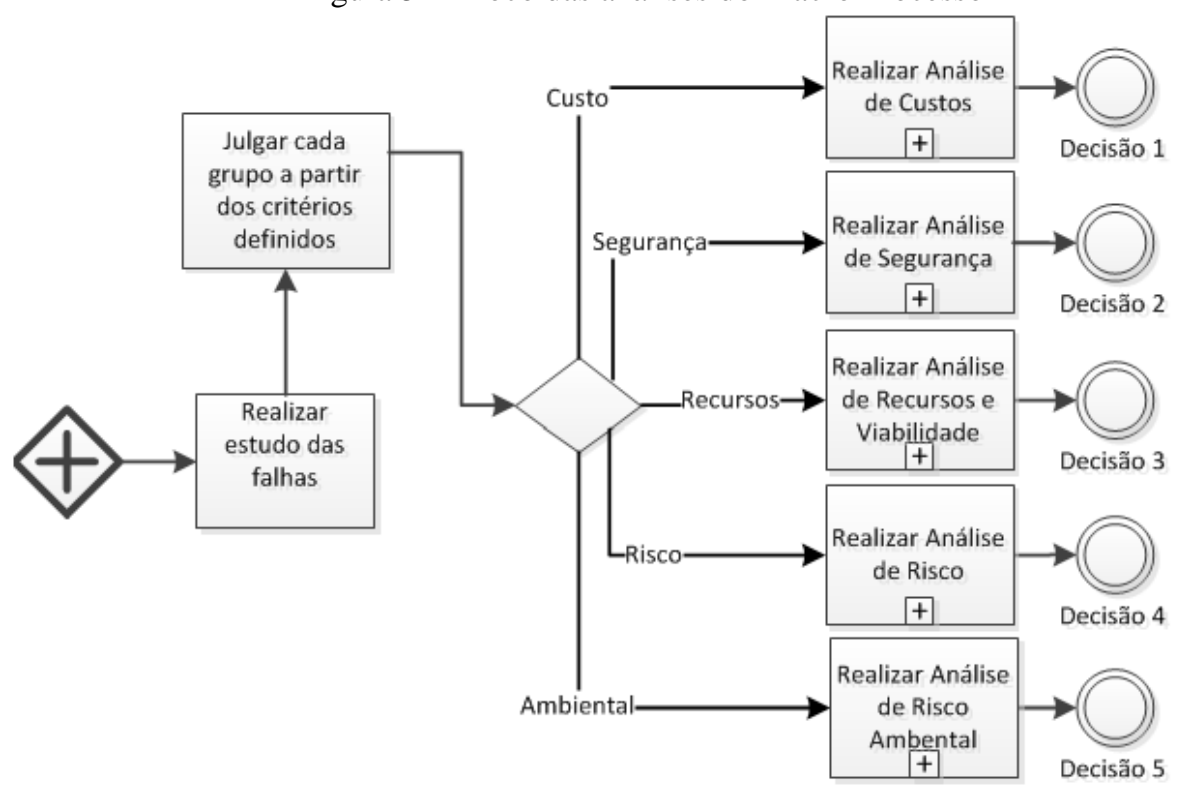

Fonte: Elaborado pelos autores

A atividade de ponderação poderá ser realizada segundo alguma ferramenta que a organização já esteja acostumada a adotar, por meio de reuniões entre os gerentes e benchmarking para decidirem qual 'peso' de relevância cada análise terá, em comparação aos demais, entre outros. A lógica do processo é obter para cada análise um valor de importância em comparação aos demais, para que posteriormente, a partir dos resultados das análises, possa obter uma decisão final. A lógica é apresentada na Tabela 2.

Os dados apresentados na Tabela 2 são simbólicos, apenas para exemplificar o procedimento. Primeiramente, elege-se o principal quesito de análise entre os apresentados (custos, segurança, recursos e viabilidade, risco e meio ambiente). A este quesito, pondera-se com o maior valor (9), para que, por comparação de relevância, obtenham-se os pesos para os demais quesitos. Esta ponderação considera alguns pontos citados por Saaty (1991) quanto à forma de comparação por pares, porém, não será formada uma matriz com os valores, como descrito para aplicação na ferramenta de tomada de decisão Analytic Hierarchy Process (AHP). 


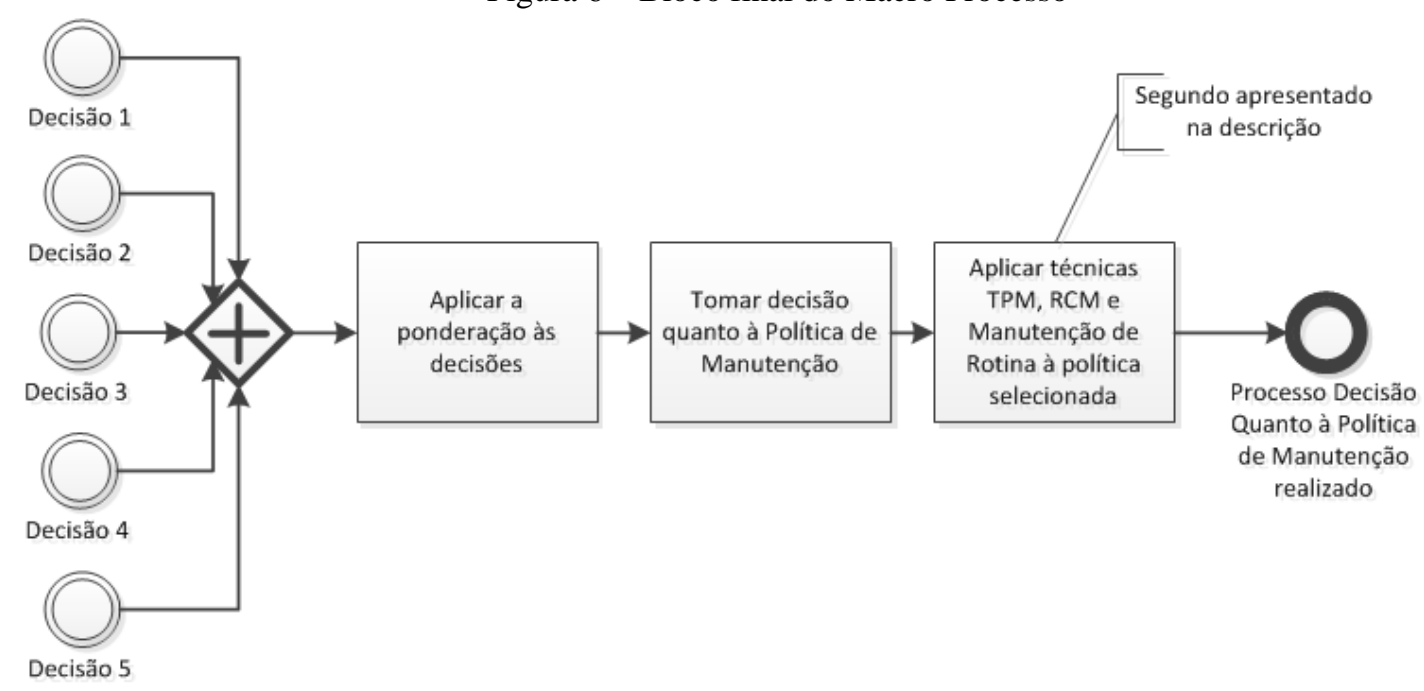

Fonte: Elaborado pelos autores

Tabela 2 - Lógica da ponderação para tomada de decisão

\begin{tabular}{l|c|c|c|cccc}
\hline & Resultado & Peso & Resultado & & Legenda & \multicolumn{2}{c}{ Total } \\
\hline Decisão 1 & MC & 9 & 9 MC & MC & Manutenção Corretiva & MC & 9 \\
Decisão 2 & MPT & 7 & 7 MPD & MP & Manutenção Preventiva & MP & 7 \\
Decisão 3 & MP & 7 & 7 MP & MPD & Manutenção Preditiva & MPD & 15 \\
Decisão 4 & MPT & 3 & 3 MPD & & & & \\
Decisão 5 & MPT & 5 & $5 \mathrm{MPD}$ & & & & \\
\hline
\end{tabular}

Fonte: Elaborado pelos autores

A partir da decisão dos 'pesos' tomada pelos envolvidos no processo (manutenção, operação e gerência organizacional), o resultado obtido para cada decisão (Manutenção Corretiva, Preventiva ou Preditiva para as cinco análises realizadas) é multiplicado pelo peso respectivo. Para finalizar, basta somar os resultados obtidos, verificando qual das políticas de manutenção obteve mais pontos. Esta política será a aplicada ao equipamento ou grupo de equipamentos analisado.

Com esta aplicação, conclui-se também a atividade 'Tomar decisão quanto à Política de Manutenção', seguindo o processo para a atividade final 'Aplicar técnicas TPM, RCM e Manutenção de Rotina à política selecionada'. Como descrito anteriormente, as políticas de manutenção mais aplicadas são as que foram utilizadas no processo, porém, diversas atividades do TPM, RCM e da Manutenção de Rotina são importantes para a aplicação de qualquer política de manutenção.

\section{Conclusão}

Trazendo uma visão holística da tomada de decisão quanto à política de manutenção, foi identificado que diversas variáveis devem ser consideradas e que existem muitas ferramentas que 
podem auxiliar na tomada de decisão, que de um modo geral é complexa e difícil. Ao longo da pesquisa, conclui-se que a hipótese de que elaboração de um processo que auxilie na tomada de decisão quanto à política de manutenção é uma ferramenta importante que pode resultar em ganhos à organização.

Todas as etapas descritas na metodologia foram seguidas a fim de atingir os objetivos específicos e o objetivo geral, resultando no processo proposto. Os pontos mais relevantes do mesmo foram a definição dos responsáveis para cada atividade e dos objetivos organizacionais, a separação dos equipamentos por ordem de importância (que influencia diretamente na política de manutenção a ser aplicada), a identificação das possíveis políticas de manutenção aplicadas (Manutenção Corretiva, Preventiva e Preditiva), as análises a serem realizadas (análise de custos, segurança, recursos e viabilidade, riscos e meio ambiente), a tomada de decisão por ponderação com base nos resultados obtidos nas análises e a aplicação de técnicas do TPM, RCM e manutenção de rotina, independente da política de manutenção selecionada.

Outro ponto importante é o fato do processo ser flexível e adaptável à realidade da organização, cabendo à mesma modificá-lo segundo suas especificidades. Com isto, conclui-se também que o processo descrito é um direcionador, levantando questionamentos e variáveis relevantes à tomada de decisão de uma forma rápida, simples e estruturada, possibilitando uma resposta com embasamento às possíveis mudanças organizacionais devido a fatores externos e internos.

Sugere-se como continuidade desta pesquisa a aplicação do processo proposto em uma empresa, quantificando os resultados, verificando as dificuldades e identificando os gaps existentes no processo. A aplicação do processo também pode ser comparada a outras ferramentas de tomada de decisão, verificando vantagens e desvantagens. Outra proposta de continuidade da pesquisa é aplicar uma ferramenta computacional à atividade de ponderação das análises realizadas, verificando os resultados obtidos. Além disto, um estudo estatístico mais amplo e aprofundado pode ser realizado para identificar outras atividades no processo, melhorando-o continuamente.

\section{Abstract}

Organizations operating in increasingly complex and changing scenarios, being influenced by external and internal factors that impact on decision-making. Among these, the definition of which maintenance policy apply to specific equipment, aiming to reduce costs and increase efficiency, is very important. From the need for a structured process to identify the most relevant variables and detail the activities to be considered, it was defined as objective to develop a process to assist in decision making as to which maintenance policy apply to certain equipment. In the proposed process were considered five analysis for decision making, including the cost analysis, security, resources and feasibility, risks and environment, each of which could result in three possible suggested maintenance policies, corrective maintenance, preventive or predictive. The research result was a macro structured process of decision-making about maintaining policy; should note that 
the proposed process should be aligned to the specificities of the organization, so it serves as a reference, raising relevant questions decision making and directing the organization.

Key-words: maintenance; process; decision-making.

\section{Referências}

ANDRADE, L; FIADEIRO, J. Agility through coordination. Information Systems, v. 7, p. 411-424, 2002. crossref

ARUNRAJ, N.S; MAITI, J. Risk-based maintenance policy selection using AHP and goal programming. Safety Science, v. 48, p.238-247, 2010. crossref

ASSOCIAÇAO BRASILEIRA DE MANUTENÇÃO. Situação da Manutenção no Brasil - Resultado 2011.

Disponível em: http://www.abraman.org.br/sidebar/documento-nacional. Acesso em: 12 dez. 2014.

BASTEN, R.J; HEIJDEN, M.C; SCHUTTEN, J.M. Practical extensions to a minimum cost flow model for level of repair analysis. European Journal of Operational Research, v. 211, p. 333-342, 2011. crossref

BERTOLINI, M; BEVILACQUA, M. A combined goal programming-AHP approach to maintenance selection problem. Reliability Engineering and System Safety, v. 91, p. 839-848, 2006. cross ref

BEN-DAYA, M. You may need RCM to enhance TPM implementation. Journal of Quality in Maintenance Engineering, v. 6, n. 2, p. 82-85, 2000. cross ref

BRIS, R; CHÂTELET, E; YALAOUI, F. New method to minimize the preventive maintenance cost of series - parallel systems. Reliability Engineering and System Safety, v. 82, p. 247-255, 2003. crossref

CHAN, F.T; et al. Implementation of total productive maintenance: A case study. International Journal of Production Economics, v. 95, p. 71-94, 2005. crossref

FACCIO, M; et al. Industrial Maintenance Policy Development: A Quantitative Framework. Disponível em <http://www.sciencedirect.com/science/article/pii/S0925527312003660 >. Acesso em: 14 dez. 2014.

FILHO, Gil. A Organização, o Planejamento e o Controle da Manutenção. 1. ed. Rio de Janeiro: Ciência Moderna, 2008.

GIL, A. Como Elaborar Projetos de Pesquisa. 5. ed. São Paulo: Atlas, 2010.

HAMMER, M; CHAMPY, J. Reengineering the Corporation: A Manifesto for Business Revolution. New York: Harper Business Books, 1993.

HASHEMIAN, H.M; BEAN, W.C. State-of-the-Art Predictive Maintenance Techniques. IEEE Transactions on Instrumentation and Measurement, v. 60, n. 1, p. 226-236, 2011. crossref

JACKSON, C; PASCUAL, R. Optimal maintenance servise contract negotiation with aging equipment. European Journal of Operational Research, v. 189, p. 387-398, 2008. crossref

KARDEC, A; NASCIF, J. Manutenção: Função Estratégica. 4. ed. Rio de Janeiro: Qualitymark, 2013.

KHAN, F; HADDARA, M. Risk-based maintenance (RBM): a quantitative approach for maintenance/inspection scheduling and planning. Journal of Loss Prevention in the Process Industries, v. 16, p. 561-573, 2003. crossref

KHAN, R. Business Process Management: a practical guide. Florida: Meghan-Kiffer Press, 2004.

KHANS, M; INGWALD, A. Common database for cost-effective improvement of maintenance performance. International Journal of Productions Economics, v. 113, p. 734-747, 2008. crossref

KIANFAR, A; KIANFAR, F. Plant function deployment via RCM and QFD. Journal of Quality in Maintenance Engineering, v. 16, n. 4, p. 354-366, 2010. crossref

LA ROSA, M; et al. APROMORE: An advanced process model repository. Expert Systems with Applications, v. 38, p. 7029-7040, 2011. crossref 
LAPA, C; PEREIRA, C; BARROS, M. A model for preventive maintenance planning by genetic algorithms based in cost and reliability. Reliability Engineering and System Safety, v. 91, p. 233-240, 2006. cross ref

LIND, S; NENONEN, S; KIVISTO-RAHNASTO, J. Safety risk assessment in industrial maintenance. Journal of Quality in Maintenance Engineering, v.14, n.2, 2008. crossref

LIU, H; LIU, L; LIU, N. Risk evaluation approaches in failure mode and effects analysis: A literature review. Expert Systems with Applications, v. 40, p. 828-838, 2013. cross ref

LOVE, P; GUNASEKARAN, A. Process reengineering: A review of enablers. International Journal of Production Economics, v. 50, p. 183-197, 1997. crossref

MARTÍNEZ, L; et al. The Graphical Analysis for Maintenance Management Method: A Quantitative Graphical Analysis to Support Maintenance Management Decision Making. Quality and Reliability Engineering International, v. 29, p.77-87, 2013. crossref

MCKONE, K; SCHROEDER, R; CUA, K. The impact of total productive maintenance practices on manufacturing performance. Journal of Operations Management, v. 19, p. 39-58, 2001. crossref

MCKONE, K; SCHROEDER, R; CUA, K. Total productive maintenance: a contextual review. Journal of Operations Management, v. 17, p. 123-144, 1999. cross ref

MUCHIRI, P; et al. Development of maintenance function performance measurement framework and indicators. International Journal of Production Economics, v. 131, p. 295-302, 2011. crossref

NAKAJIMA, S. Introduction to TPM: total productive maintenance, Cambridge MA: Productivity Press, 1988.

NGUYEN, T; YEUNG, T; CASTANIER, B. Optimal Maintenance and Replacement Decisions Under Technological Change With Consideration of Spare Parts Inventories. International Journal Production Economics, v. 143 , p. $472-$ 477, 2013. crossref

NUFFEL, D; BACKER, M. Multi-abstraction layered business process modeling. Computers in Industry, v. 63, p. 131-147, 2012. crossref

PINTELON, L; GELDERS, L. Maintenance management decision making. European Journal of Operational Research, v. 58, p. 301-317, 1992. crossref

RAUSAND, M. Reliability centered maintenance. Reliability Engineering and System Safety, v. 60, p. 121-132, 1998. cross ref

SAATY, T. Método de Análise Hierárquica. São Paulo: McGraw-Hill, Makron, 1991.

SELVIK, J; AVEN,T. A framework for reliability and risk centered maintenance. Reliability Engineering and System Safety, v. 96, p. 324-331, 2011. crossref

SLACK, N; CHAMBER, S; JOHNSTON, R. Administração da Produção. 3. ed. São Paulo: Atlas, 2009.

SUBBURAMAN, R et al. A modified FMEA approach to enhance reliability of lean systems. International Journal of Quality and Reliability Management, v. 27, n. 7, p. 832-855, 2010. crossref

SWANSON, L. Linking maintenance strategies to performance. International Journal of Production Economics, v. 70, p. 237-244, 2001. crossref

TAILLANDIER, F; SAUCE, G; BONETTO, R. Method and tools for building maintenance plan arbitration. Engineering Construction and Architectural Management, v. 18, n. 4, p. 343-362, 2011. crossref

TSAI, Y; WANG, K; TSAI, L. A study of availability-centered preventive maintenance for multi-component systems. Reliability Engineering and System Safety, v. 84, n. 3, p. 261-270, 2004. crossref

VELDMAN, J; WORTMANN, H; KLINGENBERG, W. Typology of condition Based Maintenance. Journal of Quality in Maintenance Engineering. v. 17, n. 2, p. 183-202, 2011. crossref 
VERGARA, S. Projetos e relatórios de pesquisa em administração. 9. ed. São Paulo: Atlas, 2007.

VIANA, H. Planejamento e Controle da Manutenção (PCM). 1. ed. Rio de Janeiro: Qualitymark, 2002.

WAEYENBERGH, G; PINTELON, L. A Framework for Maintenance Concept Development. International Journal Production Economics, v. 77, p. 299-313, 2002. crossref

WAEYENBERGH, G; PINTELON, L. Maintenance Concept Development: A Case Study. International Journal Production Economics, v. 89 p.395-405, 2004. crossref

WANG, L; CHU, J; WU, J. Selection of optimum maintenance strategies based on a fuzzy analytic hierarchy process. International Journal of Production Economics, v. 107, p. 151-163, 2007. crossref

WU, J; XIE, M; ADAM, T. On a general periodic preventive maintenance policy incorporating warranty contracts and system ageing losses. International Journal of Production Economics, v. 129, p. 102-110, 2011. crossref

ZHAO, Z; et al. Predictive maintenance policy based on process data. Chemometrics and Intelligent Laboratory Systems, v.103, p. 137-143, 2010. cross ref

\section{Dados dos autores}

Nome completo: Thiago de almeida rodrigues

Filiação institucional: Universidade Federal do Paraná

Departamento: Engenharia de Produção

Função ou cargo ocupado: Mestrando

Endereço completo para correspondência: Rua Comendador Corrêa Junior, 410, casa 9, Paranguá-

PR

Telefones para contato:(41) 9851-7766

e-mail: trodrigues.almeida@gmail.com

Nome completo: Robson seleme

Filiação institucional: Universidade Federal do Paraná

Departamento: Engenharia de Produção

Função ou cargo ocupado: Coordenador Mestrado/Professor

Endereço completo para correspondência: Avenida Presidente Kennedy, 1425

Telefones para contato:(41) 33613035 / (41) 91333142

e-mail: robsonseleme@hotmail.com / robsonseleme@ufpr.br

Nome completo: Marcelo Gechele Cleto

Filiação institucional: Universidade Federal do Paraná

Departamento: Engenharia de Produção

Função ou cargo ocupado: Professor

Endereço completo para correspondência: Avenida Presidente Kennedy, 1425 
Telefones para contato:(41) 33613475

e-mail:mgcleto@ufpr.br

Submetido em: 10-02-2015

Aceito em: 14-09-2015 